\title{
Germanica
}

\section{Le modernisme littéraire dans le Nord « L'art et la vie » : trois réflexions}

Der Modernismus in der nordischen Literatur Kunst und Leben: drei

Betrachtungen

Régis Boyer

\section{(2) OpenEdition}

\section{Journals}

Édition électronique

URL : http://journals.openedition.org/germanica/1500

DOI : 10.4000/germanica. 1500

ISSN : 2107-0784

\section{Éditeur}

Université de Lille

\section{Édition imprimée}

Date de publication : 1 juin 1993

Pagination : 167-182

ISSN : 0984-2632

\section{Référence électronique}

Régis Boyer, «Le modernisme littéraire dans le Nord «L'art et la vie » : trois réflexions », Germanica [En ligne], 12 | 1993, mis en ligne le 06 juillet 2012, consulté le 06 octobre 2020. URL : http://

journals.openedition.org/germanica/1500; DOI : https://doi.org/10.4000/germanica.1500

Ce document a été généré automatiquement le 6 octobre 2020.

(c) Tous droits réservés 


\title{
Le modernisme littéraire dans le Nord « L'art et la vie » : trois réflexions
}

\author{
Der Modernismus in der nordischen Literatur Kunst und Leben: drei \\ Betrachtungen
}

Régis Boyer

1 On n'entreprendra pas ici de revenir sur le bon vieux thème du «modernisme » en littérature. Il y aura bientôt plus d'un siècle, avec Baudelaire notamment, que le débat est largement ouvert et il a tant fait couler d'encre que les quelques gouttes que voici n'ajouteront rien à ce Niagara. Sur le fond, tout a été dit et l'on vient toujours trop tard! Ecrire pour son temps, exprimer la sensibilité de l'homme actuel en face des problèmes que pose l'époque présente, cela revient à justifier le bien-fondé de l'expression littéraire, en tous temps et en tous lieux et ce n'est évidemment pas pour remuer de tels poncifs que l'on propose ici les trois réflexions qui vont suivre!

Elles partent, ces réflexions, d'une sorte d'étonnement, ou d'amusement, devant la dénomination "post-modernisme» qui revient si souvent, tous ces temps-ci, sous la plume des critiques littéraires Scandinaves.Hé quoi ! Où diantre faut-il que nous nous situions si nous en sommes à une ère "post-moderne »? Sommes-nous en période de régression, ou, au moins, de stagnation, si même la dénomination "moderne » est dépassée? Admettons qu'il faille distinguer entre modernisme en général et modernisme en Scandinavie, nuance spécieuse, en vérité, surtout lorsque l'on sait quelle attention scrupuleuse, quelle fascination caractérisent les esprits du Nord, en face de tout ce qui se fait de nouveau en dehors de leur domaine géographique strict. N'oublions pas davantage à quel point ils sont ardents, ces esprits, à diviser l'histoire littéraire en branches décenniales (30-tal, 40-tal, 50-tal, etc.) et à trouver à tout prix des étiquettes à tout ce qui manifeste une quelconque originalité en regard de ce qui précédait. Il n'empêche que « post-modernisme » a, en soi, quelque chose d'insolite. 
3 Au moins pour qui n'est pas averti de l'histoire des lettres Scandinaves depuis un peu plus d'un siècle. Car il est incontestable qu'après le formidable feu d'artifices du Moyen Age, islandais en fait (eddas, scaldique, sagas et toute l'extraordinaire littérature apparentée), la Scandinavie, pendant plusieurs siècles, s'est contentée de suivre la France (âge classique, puis "Lumières»), puis l'Allemagne (Romantisme et ses séquelles). Il faudra la fantastique explosion du gennembrud, gennombrott (la "percée » moderne), à partir de Georg Brandes et de son extraordinaire magistère, en 1870 donc, pour que, tout soudain, les Scandinaves se découvrent tels qu'en euxmêmes... Encore peut-on avancer, avec quelque esprit de simplification, que c'est la révélation du naturalisme et des tendances apparentées dans d'autres branches de l'activité intellectuelle (comme le positivisme, la critique religieuse de Strauss, le darwinisme, l'utilitarisme, le marxisme à ses débuts) qui aura déchaîné un très long mouvement de remise en question dont nous ne voyons toujours pas la fin. Car en cette occurrence, il ne fait aucun doute que la Scandinavie a décidé, plus ou moins consciemment, de sortir d'habitudes fort anciennes pour entrer de plein pied dans la problématique $\mathrm{du}$, de notre modernisme. On peut donc admettre que "postmodernisme » s'applique à un état de choses où les apports du gennombrott auront été assimilés puis dépassés - ce qui reviendrait, donc, à prendre le « modernisme » comme quelque chose de daté et de passé.

4 Mais non sans nombreuses retombées un peu dispersées. Suivons les spécialistes qui entendent mettre en relief les quatre grands «intercesseurs » de notre modernisme. Soit Rimbaud acceptant la faillite de l'art traditionnel à exprimer les désarrois de la conscience moderne, Mallarmé vivant le drame de récriture impossible et cherchant avec une application comme maniaque le terrain d'élection d'un nouvel espace littéraire, Lautréamont faisant de la parole même la matière de l'écriture et Flaubert appliqué à faire éclater, par la répétition forcenée, la force du lieu commun. Il reste que, vers le dernier quart du XIXe siècle, non seulement il n'est plus possible d'écrire comme on le faisait auparavant, mais encore, que tout ce qui pouvait passer pour incitation recevable à effusions littéraires est refusé, voire gravement contesté : on ne soulignera jamais assez, à cet égard, le rôle déterminant de S. Kierkegaard qui fut et reste, par excellence, le pré-gennombrudsmand. Il y avait, chez lui puis chez ses épigones, un antitraditionnalisme, un anticonventionnalisme qui vont demeurer la règle de quiconque entend, dans le Nord, suivre son temps.

5 Le fait est d'autant plus remarquable que nous savons tous le poids contraignant de la tradition, notamment en matière d'expression littéraire, dans les pays du Nord. Qu'une poignée d'écrivains de génie aient pu, en quelques décennies, rompre en visière avec ce qu'il était convenable de dire, avec la façon reçue de le dire, c'est certainement un fait difficilement acceptable chez nous qui vivons régulièrement, depuis des siècles, la querelle des Anciens et des Modernes, mais d'évidence, c'est de là qu'il faut partir pour accepter ce « post-modernisme » qui nous retient tant ici.

6 En d'autres termes: devons-nous entendre par "post-modernisme » tout ce qui, ayant liquidé les apports révolutionnaires de la "percée moderne ", s'est engagé dans des voies encore plus iconoclastes? J'aimerais répondre par l'affirmative : postuler que le gennembrud est maintenant acquis définitivement, que toutes ses retombées sont acquises, admises, qu'en conséquence, la question n'est plus d'aller dans le sens des fracassantes remises en question d'un Ibsen, d'un Strindberg, d'un Jacobsen - entre 
autres - mais bien de voir vers où faire porter les faisceaux lumineux d'un projecteur inlassable.

7 C'est-à-dire tenons pour admis tous les mots d'ordre des gen-nembrudsmaend, considérons que le "modernisme " qu'ils représentèrent avec éclat est désormais partie intégrante du patrimoinelittéraire Scandinave.Qu'y a-t-il au-delà ? Et - c'est une préoccupation qui ne m'a jamais laissé en repos depuis plusieurs décennies que je m'emploie à diffuser ces littératures - que faut-il entendre par modernisme à l'heure actuelle dans le Nord? Comme je l'ai dit en commençant, je n'aurai pas la présomption de répondre avec assurance, voici seulement trois réflexions que j'aimerais proposer au lecteur en le priant de les tenir pour des ouvertures ou des hypothèses plutôt que pour des certitudes!

La première réflexion revient à une constatation de type banal: le monde a prodigieusement changé en un demi-siècle, et, sans doute, beaucoup plus pour le Nord que pour le reste de l'Occident. Y a-t-il, tout bien pesé, une véritable continuité entre le Varmland de Selma Lagerlöf et le Södermannland de Sven Delblanc (je prends à dessein deux écrivains suédois du type «traditionnel »), entre le Nordland des handelsteder de Knut Hamsun et l'Oslo de Jan Kjaerstad (deux romanciers norvégiens qui, pourtant, tiennent à rester fidèles à une certaine façon d'écrire), voire entre le Sjaelland de certaines nouvelles de Karen Blixen et la Copenhague de Tage Skou-Hansen (qui tiennent toutefois, l'un et l'autre, à narrer des « histoires ») ? Et je ne parviens pas à évaluer quelle serait la réaction d'un Einar Benediktsson devant un « roman » comme Kristnihald undir Jôkli (Úa ou Chrétiens du glacier, dans la traduction française) de Halldor Laxness! Comme le dit, en substance, la Tante Disa dans Ljugarstriden (1956) d'Arne Sand, il n'y a plus moyen de mettre un peu d'ordre dans le tourbillon du monde actuel... Et mettre de l'ordre n'a-t-il pas toujours été le souci constant de tout bon Scandinave?

9 De là, comme s'expriment les Danois, cette indignationslitteratur, ou cette debattlitteratur qui auront joui d'une telle faveur, là-bas, depuis plusieurs décennies. Nous nous amusons de constater qu'en face de toute difficulté nouvelle, dans quelque domaine que ce soit, les Suédois s'attachent premièrement à mettre en place un utredningskommitté chargé de résoudre le problème après concertation! Soit! Mais à partir de quelles bases intouchables, ou en fonction de quelles nouvelles données incontournables? Rien d'étonnant à ce que, dans le Nord, les événements de 1968 aient été pris avec un sérieux bien plus grand qu'ailleurs. Ces sociétés tradi-tionnalistes restées par définition bien plus solidement attachées à leur passé agraire ou rural que sous nos latitudes, fermement enracinées dans des valeurs « de la terre » qu'a dûment étudiées le numéro 4 (1988) de la présente revue, projetées, en quelque sorte, dans notre ère industrielle avec toutes ses conséquences, et qui ont tenu à jouer consciencieusement le jeu, ne peuvent, à leurs moments de lucidité, que s'interroger devant la manière de chaos qu'offre aux regards la conjoncture! Etre moderne, cela revient en première approximation, à oser dire ce que l'on n'a encore jamais exprimé, peut-être par puritanisme (cette dimension ne doit jamais échapper à l'observateur, serait-elle prise dans son acception inverse, il y a un puritanisme de l'antipuritanisme, la «brutalité » d'un Agnar Mykle, pour ne prendre que ce cas, n'appelle sans doute pas d'autre explication), peut-être par une peur légitime (les attitudes d'un P.C. Jersild 
pourraient relever de cette analyse), peut-être surtout par une reculade somme toute compréhensible devant une vision du monde où le vide tend à abolir une prétendue solidité de type religieux qu'aucun aggiornamento opportun n'est venu combler - il faudrait évoquer ici celui qui, à n'en pas douter, reste le directeur de conscience de toute la génération présente, Stig Dagerman!

10 Tant il est clair que l'on ne peut plus ni écrire, ni même lire comme naguère. Qui veut être entendu, il faut qu'il sache à qui parler, et comment. Or, que lui présente le spectacle du monde ? À quel homme faut-il s'adresser ? À cet être désorienté que nous dépeint Peter Seeberg ? À cet errant kafkaïen que s'est attaché à nous décrire Villy Sørensen pour qui toutes les valeurs semblent s'être définitivement dissoutes ? À cet être de désordre et d'incohérence que nous livrent aussi bien les manières de confessions d'un Jan Myrdal que les implacables accusations d'un Thorkild Hansen? À cet anormal que dissèque d'un scalpel d'expert un Stig Larsson? Ou, pour en rester là, à cet individu fragmentaire, miné intérieurement, que pressentait déjà Pär Lagerkvist et que stigmatise Lars Gustafsson? Quand on aura multiplié les images déchirantes, les effets de surprise plus ou moins tératologiques, à la Lars Gyllensten, quand on se sera fixé pour tâche de dépoétiser toute expérience créatrice et de substituer des montages par définition artificiels au donné brut de l'expérience - je pense, bien entendu, aux recherches d'un Sven Holm ou d'un Sven-Age Madsen - qu'aura-t-on atteint? On a voulu à toute fin identifier modernité et nouveauté, liberté individuelle, recherche de traits spécifiques: fort bien! Pour ces tempéraments répugnant aux excès du fantastique tout comme aux élucubrations purement abstraites, la bonne vieille interrogation bernanosienne se pose avec une redoutable acuité : la liberté, pour quoi faire ? Le cas - j'en évoquerai d'autres, mais celui-ci vient fort bien ici - de Dag Solstad qui renonce à son marxisme fracassant et à son nynorsk pour revenir, avec Gymnaslaerer Pedersens beretning om den store politiske vekkelsen som har hjemsøkt vart land (1982) à des considérations plus " humaines ", plus conformes à l'idiosyncrasie de son peuple, le tout en bokmål, me paraît éclairant. On sait bien que le radicalisme, comme on dit là-bas, joint à ce qu'il faut bien appeler une certaine naïveté poussent les meilleurs esprits du Nord à essayer d'aller jusqu'au bout d'une tentative nouvelle, à partir du moment où elle parait recevable. Mais la conscience sourde de certaines limites à ne pas outrepasser n'abdique jamais, un peu comme dans ces nouvelles paysannes de notre Marcel Aymé où un conflit local et banal se met à dégénérer jusqu'à prendre des proportions catastrophiques que les plus lucides, les plus équilibrés entrevoient à temps pour arrêter les dégâts. Comment expliquer autrement l'étonnant succès, tout à fait actuel, de talents bien peu «modernistes » comme ceux de Torgny Lindgren ou de Göran Tunström ? Entre autres...

11 Évidemment, l'histoire, la politique surtout, n'ont rien fait pour tempérer les audaces et les rages destructrices. Et, dans des pays sensibles à l'actualité comme le sont les Scandinaves,il ne faut jamais faire abstraction de cet élément. De nouveau, je n'entreprendrai pas, ni de faire le procès de notre époque, ni même d'en proposer une sorte d'analyse spectrale. Il est clair, tout de même, que les assises mêmes de notre société traditionnelle sont en train de s'effondrer. Pour ne donner qu'un exemple, le modernisme est à un féminisme égalitaire et triomphal devant lequel reculent plus ou moins des mentalités plus «méridionales». Mais, dans la ligne précise du radicalisme que je viens d'évoquer, on ne peut admettre autrement des œuvres comme celles d'Ulla Isaksson ou de Svává Jakobsdóttir. Ou bien, dans le domaine strictement politique, les apports des "prolétaires» suédois, danois, norvégiens sont en passe de devenir des 
acquis tout comme la social-démocratie qui s'est faite avec eux sinon grâce à eux. Toutefois, sans revenir sur Dag Solstad ni même en référer à l'actualité, suédoise notamment, la plus brûlante, il parait tentant de considérer qu'une sorte de "virage à droite " (à l'échelle de ces pays) est en train de s'opérer sous nos yeux. A telle enseigne que des efforts comme ceux que tentèrent les revues norvégiennes Profil, après Mot Dag paraissent devoir, désormais, relever de l'histoire littéraire plus que de l'actualité !

Il en va de même en psychologie, où les Scandinaves sont allés fort loin, sans doute sur des modèles américains, mais il n'importe pas tellement ici. Lars Gyllensten se demandait dès les débuts de son œuvre comment mener cette vie courte et désagréable qui nous est octroyée dans le monde absurde et cruel que nous hantons. Il a fait école : c'est un jeu un peu gratuit et assurément sadique, chez des peuples qui ont toujours tenu la santé et l'équilibre pour des valeurs sûres, que de recenser toutes les œuvres où l'on met en scène des anormaux, des cas cliniques, des déviants. L'intention est certainement bonne, il s'agit de ne pas négliger les laisser pour comptes de nos sociétés, mais l'impression retirée ne manque guère de laisser un goût de malsain ou de pathologique, même quand, comme c'est le cas chez Göran Tunström, notamment dans l'admirable Oratorio de Noël (1983), l'étude appliquée du psycho-tisme revient à une exaltation de la vie. C'est bien, d'ailleurs, ce que voulait dire, déjà, Sven Fagerberg dans ses nombreux romans quand il tenait à chanter det växande, c'est-à-dire la part intuitive, l'instinct de vouloir-vivre vivace qui se refuse à abdiquer devant la machine et la société de consommation.

Je répète qu'il y a là, au-delà d'une volonté de sacrifier et aux modes ambiantes et à une contemplation comme objective du monde actuel, un refus, en dernière analyse, devant les inévitables excès, admissibles dans des littératures où probablement, la chose littéraire est moins prise au pied de la lettre que dans le Nord, mais, en soi, proprement « invivables ». Prenez ce culte de la dispersion, cette sorte de jubilation artificielle mais source de jolies constructions mentales, que l'on peut trouver dans l'œuvre d'un Georges Bataille et qui finit par déboucher sur le silence, bien littéraire, d'un Samuel Becket. Je ne leur trouve pas d'équivalents au-delà du Danevirke. Ou encore : le refus de la linéarité et de l'à-plat du texte avec tentative de création d'un nouveau rapport entre le temps et l'espace de l'écriture dont on pourrait trouver un exemple chez Jacques Derrida, sous la forme de littérature-calligramme. J'en cherche vainement trace, même au pays de Gunnar Ekelöf ou à celui de Sigurdur Pálsson.

14 Exprimer un nouvel être humain... Qui serait conforme aux exigences d'un monde «moderne ». D'accord, mais si ce monde-là ne pouvait réellement pas exiger de l'homme qu'il se plie à ses lois? Car il s'appelle machines, techniques, informatique, etc. Et n'est-il pas vrai que les extases enfantines d'un Andersen devant les merveilles de l'Exposition Universelle de Paris, en 1867, ne sont plus de mode aujourd'hui ? Ces pays-là, tout compte fait, sont allés plus loin que nous dans tous ces domaines, on ne le contestera pas. C'est anticiper sur mes conclusions, mais ne se pourrait-il pas que leur modernisme soit plus conscient que le nôtre des bornes à ne pas outrepasser et qu'en conséquence, il soit en train, maintenant, sous nos yeux, de faire pour ainsi dire la part du feu ? C'est, en tout cas, la première réflexion que m'inspire le sujet. 
La seconde sera d'ordre plus technique. On perd beaucoup si l'on oublie que, dans le Nord, modernisme s'entend toujours, en première analyse, sur le plan formel, tant la tradition est forte là-bas. Je note, par exemple, qu'Edith Södergran, dont il m'est arrivé de m'occuper, fut d'abord récusée pour avoir violé les saintes lois de l'écriture poétique, ou que les poètes « atomistes » islandais des années 1950 furent répudiés par la «bonne» critique qui leur infligea l'épithète en question (somme toute fort bien venue tant leurs poèmes, dans leur forme ramassée, s'entendent à déchaîner des réactions en chaîne) parce que leur façon de s'exprimer menait au chaos la langue islandaise comme venait de le faire du sol de Hiroshima la fameuse bombe américaine. Que l'une, comme les autres, aient traité des thèmes qui n'étaient pas réputés "poétiques" à l'époque mais coïncidaient avec la vision de leur modernisme réciproque, ce n'était pas cela qui avait d'abord retenu l'attention sourcilleuse des aristarques locaux. Quand des "concrétistes" comme Rolf Jacobsen, Norvégien, ou Øyvind Fahlström, Suédois, chantent le béton, le fer, la pierre brute etc., quand des «travailleurs sociaux » comme P.O. Sundman cherchent à appliquer à la littérature les principes de leur métier, ce n'est pas d'abord sur le fond qu'ils sont jugés, mais bien sur la forme et je gage que les incessantes répétitions d'un Stig Claesson ont, d'abord, plus nuit à la réputation de cet écrivain important que ce qu'il entendait signifier en profondeur.

16 Sans doute, là encore, faut-il prendre en compte les influences venues de l'étranger. Conformément aux vues qui ont été suggérées plus haut, ces ruptures formelles épousent les désarrois d'une vision du monde désaccordée avec les usages immémoriaux. De là vient que, dans le Nord comme ailleurs, le phénomène immédiatement perceptible en matière de modernisme littéraire tient à l'éclatement de la forme typiquement moderne de l'écriture : le roman. Éclatement qui n'est ni plus ni moins visible ou poussé qu'ailleurs, mais qui, sans doute, est davantage ressenti parce que la tradition conteuse aura certainement été bien plus forte aux pays des sagas ou des contes populaires qu'ailleurs. On aura prêté une attention extrêmement active, dans le Nord, à l'observation patiente des tropismes individuels ou collectifs, chère à Nathalie Sarraute - je pense à P.O. Enquist - ou à cette sorte d'arpentage frénétique typique de Robbe-Grillet : prenez le cas de Lars Gustafsson.

17 Pareillement, la volonté s'est clairement manifestée de mélanger toutes les sortes d'art, tous les styles, aux mêmes fins que partout ailleurs : découvrir une langue nouvelle qui vaudrait pour l'homme d'aujourd'hui. Il y aurait, au moins, à évoquer ici le chefd'œuvre de Per Hultberg, Requiem (1985) avec ses cinq cent trente-sept monologues d'autant de personnages différents au moment précis où chacun sort de sa crise personnelle pour tenter de la dire et, donc, de l'objectiver. Parlons de "déconstructivisme" puisque cette étiquette-là n'a pas laissé insensible la critique férue de dénominations, puis de reconstructivisme. Qu'importe? Il reste que tout écrivain Scandinave sérieux est infiniment conscient des ressources de sa langue et, en bon artisan qu'il est par définition, s'applique à la travailler sans cesse pour l'amener à dire exactement ce qu'il ressent. En somme, rien de nouveau pour un amateur des prouesses techniques de l'âge viking ou des stavkirker.

18 De nouveau, mais l'insistance n'est pas superflue, nous sommes en terrain internationalement connu: de telles recherches ne déparent pas à l'âge de la musique sérielle, de la peinture de collages, de l'architecture gratuite, tous domaines où les Scandinaves ont fourni des témoignages de valeur. Comme le dit Rolf Jacobsen : «Le 
poète est de ceux qui voient la poésie comme une sorte de bras prolongé du langage [...]. Le poème commence, pense-t-il, là où les journaux finissent. Il s'efforce de prolonger les lignes un peu plus loin. Comme la musique, comme la peinture, comme le langage des signes mathématiques, il essaie d'élargir notre connaissance, notre horizon et nos protestations jusqu'à des domaines où les alphabets quotidiens n'atteignent pas » (préface à Choix, 1977). On comprend également l'intérêt, très vif dans le Nord, qu'auront porté à peu près tous les grands écrivains à ces formes neuves d'expression que représentent le théâtre radiophonique, la télévision, le film, le quotidien, tous véhicules à l'emploi desquels nos délicats ne condescendent pas toujours! C'est surtout en poésie, et de préférence dans le domaine lyrique, que cette tendance se sera manifestée le plus clairement. Déjà bien avant la période qui nous intéresse ici, et à l'évidence dans le sillage des tentatives dadaïste et surréaliste françaises, un Björling, un Lindegren et, plus grand que tous, un Ekelöf avaient tendu vers une poésie insaisissable selon les crochets de l'analyse tradition-nelle, mais aussi proche que possible de la musique, voire de tout moyen d'expression que l'homme moderne, consciemment ou non, tendait à substituer aux voies (aux voix) traditionnelles.

On peut faire des remarques similaires à propos d'autres types d'approche, comme ce " néo-simplicisme » (puisqu'il faut à tout prix des étiquettes) de Göran Sonnevi : il veut dire «brutalement» le spectacle du monde, Vietman à l'appui, et en conséquence, s'exprimer, en poésie, en termes quotidiens, vulgaires, grossiers d'aventure, bref, "dépoétiser» la poésie. Encore une fois, cela ne choque pas réellement au pays de Prévert ou de Jean Genêt, tenons que c'est une gageure hasardeuse vis-à-vis des compatriotes de Cari Snoilsy !

Le terme va de soi, en quelque sorte et, en vérité, il faut le tenir plus pour un ultime refuge que pour une façon décisive de trancher le débat. Ici comme ailleurs, le langage a fini par devenir une fin en soi et si, dans le domaine strict où nous sommes depuis le début de ce développement, l'on doit parler de modernisme, c'est probablement là qu'il se situe. J'en prends la formulation précise chez Kerstin Ekman (dans En stad av ljus) :

Il n'y a que moi ici. Il n'y a que moi, le papier, la pointe de la plume, l'encre. Je change. A chaque mot, je me modifie. Un alphabet se coule hors de moi, il bouge sur le papier comme des animalcules sur le lit d'une rivière. Je me coule hors d'un alphabet, je ne suis que mouvements, réflexes sur un fond de sable, je suis les mouvements de l'eau et les éclairs de lumière. La pointe de la plume m'écrit. Je pousse cet écrit sur le papier. Non, l'encre m'écrit comme pluie, comme feu d'herbes sèches sur les côtés. Elle laisse un écrit en moi, des traces dans ma chair, des cicatrices emplies d'encre. Ce n'est que moi, que la pointe de la plume qui bouge. Écrit.

21 Expérience commune de nos jours, certes, mais qu'il me paraît important de trouver sous une plume Scandinave. Car j'ai toujours tenu que le fait d'écrire, d'écrire pour raconter, de raconter tout court résume le tout de l'inspiration, dans le Nord. Quelque chose me pousse à mettre la longue citation qui vient d'être faite en parallèle exact avec ce passage bien connu Ď August, de Knut Hamsun, où il est dit en substance, à propos de ce maître hâbleur et fabulateur qu'est August: Raconte, August, raconte, nous savons bien que tu dis des mensonges, mais tu parles comme un journal, raconte encore...

Et c'est ce qu'a fort bien vu, senti Inger Christensen, qui me paraît avoir poussé le plus loin cet aspect du modernisme en littérature. Elle écrit, dans Det (c'est le poème $\mathrm{n}^{\circ} 1$ de « La scène ») : 


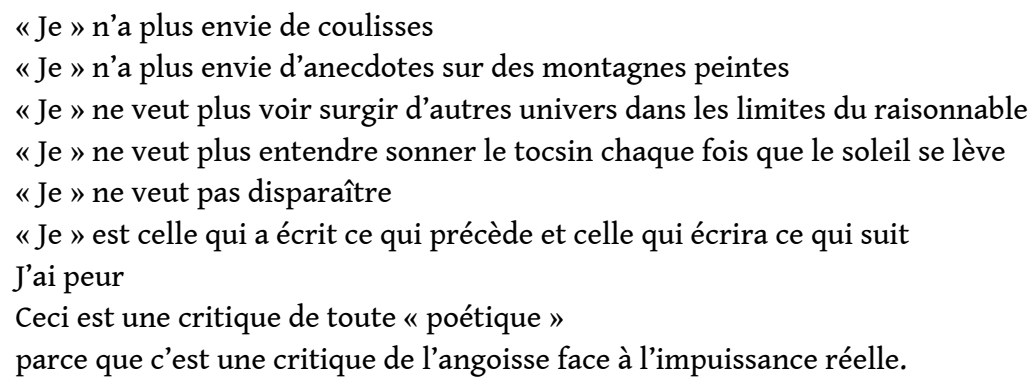

Le procès me paraît instruit. C'est uniquement pour être complet et rester aligné jusqu'au bout sur les goûts du jour que je note encore que, à cet égard, les courants derniers-nés de la littérature occidentale ne sont, bien entendu, pas absents de ces recherches Scandinaves en matière de modernisme. Il s'agit d'échappées dans le mythe, dans la symbolique ou dans la sémiotique (cette dernière déjà bien présente dans les essais d'Inger Christensen) qui, ici comme ailleurs, encore une fois, tentent de sauver l'essentiel en récupérant ce qui ne saurait périr. La fortune du "roman-document » qu'ont illustrée tant d'écrivains depuis Sara Lidman et où Dorrit Willumsen tient une place de choix (avec Marie, 1983), celle du faux roman de science-fiction, à la Kjartan Flögstad (U3), celle du prétendu roman biblique de Torgny Lindgren (Bethsabée, 1984) relèvent si clairement de cette soif de dépasser les contingences pour retrouver les sources vives que l'on rougirait d'insister.

Sources vives : ce sera mon dernier thème, bref celui-là, de réflexion. La lecture des pages qui précèdent aura convaincu, je le souhaite, du fait que rien de ce qui est « moderniste » n'a été étranger aux Scandinaves, en littérature (et certainement aussi en art tout court, mais tel n'était pas l'objet du présent essai) depuis un bon nombre de décennies. Ils ont vécu, plus intensément peut-être, plus rapidement et radicalement, à coup sûr, les heurs et malheurs de notre condition présente. À cet égard comme à tant d'autres, on gagne toujours à étudier attentivement ce microcosme qu'est le Nord car il nous offre une sorte de raccourci de nos problèmes et des solutions que nous tentons de leur apporter, qui risque souvent de nous échapper au sein de communautés plus vastes, moins bien centrées et davantage victimes des aléas de l'Histoire. Exemplaires à plus d'un titre, ces Scandinaves, ne serait-ce qu'au titre des présentes réflexions. Redisons-le : ils ont joué le jeu, ils n'ont pas essayé de s'en soustraire au nom de je ne sais quel isolationnisme, périmé de toute manière. Donc, en matière de modernisme, fond et forme, ils ont vaillamment exploré toutes les voies qui s'offraient, avec un bonheur inégal, mais honnêtement, l'adverbe qui leur convient par excellence, je crois.

Dirai-je qu'à l'heure actuelle, ils me semblent avoir atteint ce point, que j'ai évoqué plusieurs fois à divers titres, non pas de non retour, mais de saturation.

Car, lorsque l'on est homme du $\mathrm{XX}^{\mathrm{e}}$ siècle, et, en particulier, lorsque l'on est Scandinave, il semble qu'il y a des invariants dont on ne peut faire abstraction. Va pour les derniers trains de la mode: on a toujours tellement peur, dans le Nord, d'être demeuré " provincial », de ne pas pouvoir faire pièce au dernier-né de la mode anglosaxonne, allemande, sud-américaine ou française. Mais il reste des limites imprescriptibles. Et l'on voit reparaître - ou même sous-tendre comme innocemment les recherches les plus audacieuses - de bons vieux thèmes impérissables qui semblent 
ne jamais devoir mourir. Je n'en établirai pas la liste car elle serait longue et, au demeurant, fastidieuse, voire bien connue du spécialiste. Mais notez comme le motif religieux, hérité de cinq siècles de luthéranisme sinon d'antiques assises mi-païennes, mi-sociologiques, de la faute et de la culpabilité, de la responsabilité afférente, soustend des œuvres comme celles de la socialiste Sara Lidman ou de l'« existentialiste " Stig Dagerman. Remarquez comme les immortels tropismes "naturels", la fleur, l'oiseau, le lac, la forêt, l'amour, la mort, la vie servent, à tout coup, de trame à toute œuvre littéraire, quel qu'en soit le prétexte anecdotique. Je n'en veux pour exemple que l'œuvre de Tarjei Vesaas, qui fut tellement sensible aux vents destructeurs nés du surréalisme et de la psychanalyse et qui, malgré tout cela, nous aura laissé plus qu'un chef-d'œuvre, un véritable message, avec Les oiseaux. Et l'on pourrait prodiguer à l'infini les cas d'écrivains "modernistes" totalement engagés dans des recherches dont on vient de donner quelque idée mais qui, ou bien n'ont jamais cessé de rester fidèles à une inspiration atemporelle, ou bien sont revenus à cette source après un temps d'errances.

Car il reste, en dernier ressort, à revenir sur un point qui a déjà été abordé plusieurs fois ici. Depuis qu'ils écrivent, les Scandinaves n'ont jamais cessé un seul instant d'être fascinés par la belle histoire d'amour et de mort dont il reste vrai de dire, comme Thomas au début de son Tristran, que rien ne saurait nous plaire davantage. Les trois plus forts ouvrages que j'ai lus ces dernières années - bien entendu, la subjectivité du choix ne peut échapper au lecteur, mais j'entends l'assumer pleinement - s'intitulent Gheel, la ville des fous, de Per Odensten (1981), Gobi, de Tor-Åge Bringsvaerd (1985) et Requiem, déjà cité, de Per Hultberg (1985). Est-ce un hasard si ce sont, tous les trois, de fabuleux récits, agencés avec tout l'art que l'on voudra, selon toutes les formules modernistes possibles, mais des récits, des narrations, de grandes et belles histoires qui disent cent fois plus la rage de vivre que les mornes délectations du néant ? À mes yeux, c'est avant tout cela, le modernisme dans les pays du Nord, en matière de littérature : prendre en compte les incitations du moment, assurément, mais sans réellement rien sacrifier d'essentiel à l'art de dire. Parce que, pour mille raisons qu'il n'est pas utile de détailler ici, la volonté de vivre, immémorialement, a toujours été plus forte dans le Nord que le culte, bien littéraire d'ordinaire, des affres du lendemain incertain. Art, soit, art moderne si l'on y tient puisque telle est la règle du jeu, mais non au détriment de la vie, serait-elle un peu brute, un peu " primitive ».

Je ne refermerai pas ce petit dossier sans avoir cité à la barre deux témoins de choix encore.

Le premier est Danois et s'appelle Klaus Rifbjerg. On peut dire de lui qu'il n'est pas de voie moderne, moderniste qu'il n'ait vaillamment explorée : il s'est exercé dans à peu près tous les genres imaginables, poésie, roman, essai, relations de voyages, scénarios de films, émissions de radio, séries télévisées, théâtre, collages, journalisme, et partout, en s'efforçant de renouveler ce qui se faisait avant lui. C'est d'ailleurs ce qu'a parfaitement vu le Conseil Nordique lorsqu'il lui a attribué son enviable prix, en 1970 déjà : « dans tous les genres où il s'est exercé, il est parvenu à brosser avec succès un portrait original de son époque ». Mais à travers toutes ses fantaisies, tous ses tours de prestidigitation, il demeure un regard d'enfant aimant passionnément la vie. En fait, on résumerait correctement cette inspiration, telle qu'elle s'exprime, par exemple, dans 
Mythologie, en disant qu'elle est demeurée, de bout en bout, une méditation sur la responsabilité de l'homme moderne en face de cette vie que l'on a défigurée, fossilisée, et qu'elle revient à un hymne discret mais intense à la vie radieuse que les forces occultes ou mauvaises de la bêtise actuelle cherchent à tuer.

Ou bien prenez l'Islandais Thor Vilhjálmsson. Son cas est encore plus explicite car on chercherait en vain un adepte plus fervent de tout ce que la mode, le modernisme ont inculqué aux esprits modernes : il n'a pas d'équivalent, dans son pays, en fait de diffuseur, d'initiateur, d'intercesseur; depuis quarante ans, il n'est pas de courant nouveau, en art, en philosophie, dans les lettres qu'il n'ait vulgarisé et défendu avec une fougue sans égale. Et lui-même s'est appliqué à écrire des ouvrages, poèmes, essais, romans, pièces de théâtre auxquels il est dérisoirement facile d'accoler toutes les caractérisations en -isme qu'aura prodiguées notre temps. Et puis soudain, en 1986, à soixante ans passés, il décide de revenir à ses sources islandaises et il nous offre un superbe roman (qui lui vaudra, ipso facto si je peux dire, le prix du Conseil Nordique) intitulé La mousse grise brûle, où il renoue avec $\mathrm{F}$ antique tradition de ses ancêtres sagnamenn (auteurs de sagas). Et où, sur des données historiques vraies, à partir d'un de ses compatriotes qui a réellement existé, il renoue avec cette exaltation du vouloirvivre, de l'amour de vivre qui a toujours été la marque d'un authentique Scandinave. Superbes retrouvailles de l'art et de la vie!

\section{RÉSUMÉS}

L'essai de Régis Boyer se présente sous la forme de "trois réflexions " sur le modernisme Scandinave en littérature. Il souligne d'abord l'ambiguïté du terme « modernisme » et constate, à partir de la dénomination, pour lui aberrante, mais courante en Scandinavie, de "postmodernisme » que le véritable modernisme Scandinave reste attaché au gennembrud (1870) et à ses séquelles. En somme, les gennembrudsmaend sont ceux qui ont osé dire ce que leurs contemporains, trop longtemps attachés aux courants venus de France ou d'Allemagne, n'avaient pas eu l'audace d'exprimer.

Sa première réflexion porte sur les thèmes du modernisme. Ils n'ont rien de particulièrement original, ils s'attachent, là comme partout ailleurs en Occident, à coïncider avec les changements radicaux intervenus, dans tous les domaines, dans un monde en mutation radicale. L'accent est porté sur des modifications profondes qu'a subies la vision de l'homme, de la vie et du monde dans un univers qui aura subi une évolution plus radicale et, peut-être, plus profonde qu'ailleurs. Mais en somme, l'originalité du modernisme Scandinave, sous cet angle, ne lui paraît pas déterminante.

C'est pourquoi sa seconde réflexion est plus attentive : elle concerne les innovations techniques, formelles qu'a apportées le Nord en matière de littérature. Un éclairage plus vif est porté sur celles de ces modifications qui marquent une rupture plus évidente avec des modes d'expression traditionnalistes.

Mais, et c'est sa troisième réflexion, la nouveauté, pour chère qu'elle soit, ne va pas, dans le Nord, au-delà de limites «raisonnables", c'est-à-dire que des valeurs immémoriales paraissent triompher de tous les vents de la mode. Une certaine échelle de valeurs morales et religieuses, 
une défiance des abstractions gratuites et surtout une fidélité comme inconditionnelle à l'art de raconter lui paraissent marquer les limites du modernisme Scandinave tout en sauvegardant ce qui, de toujours, a fait la spécificité de ces inspirations.

Der vorliegende Essay beschäftigt sich mit dem skandinavischen Modernismus indem er drei verschiedene Gebiete in Betracht zieht. Zuerst wird die Zweideutigkeit des Wortes «Modernismus» unterstrichen und darauf hingewiesen, daß der wirkliche Modernismus, trotz der in Skandinavien geläufigen aber irrigen Bezeichnung von «Post-Modernismus », eng mit dem gennembrud und seinen Ausläufern verbunden ist. Die gennembrudsmaend sind schliesslich diejenigen die es gewagt haben, Öffentlich zu bekennen, was ihre Zeitgenossen, die zu lange an die aus Frankreich oder Deutschland kommenden Strömungen gebunden waren, sich nicht zu sagen trauten.

Die erste Betrachtung bezieht sich auf die Themen des Modernismus. Sie weisen keine besondere Originalität auf und stehen sichtlich im Zusammenhang, wie sonst überall in Europa, mit den radikalen Veränderungen, die auf allen Gebieten einer in radikaler Wandlung stehenden Welt erschienen sind. Es wird besonders Nachdruck gelegt auf die tief einschneidende Modifizierung des Menschenbildes, der Lebens- und Weltanschauung in einer historisch gegebenen Umwelt, deren Entwicklung vielleicht tiefer und radikaler war als sonstwo. So betrachtet erscheint allerdings die Originalität des skandinavischen Modernismus nicht besonders ausschlaggehend zu sein.

Die zweite Betrachtung beschäftigt sich darum eingehender mit den technischen, formellen Neuerungen, die der Norden auf dem Gebiet der Literatur gezeitigt hat. Näher beleuchtet werden diejenigen unter diesen Neuerungen, welche einen klareren, eindeutlicheren Bruch mit den traditionnellen Ausdrucksformen bedeuten.

Die Neuerung aber - und darin Hegt die dritte Betrachtungslinie - geht im Norden nie über «vernünftig » erscheinende Grenzen hinaus, so sehr man auch daran hält; dies bedeutet also, daß althergebrachte Werte, allen Winden der Mode zum Trotz, sich siegreich durchfechten. Eine gewisse ethische und religiöse Wertskala, ein Misstrauen vor sich selbst genügenden Abstraktionen und besonders ein fast bedingungsloses Festhalten an der Kunst der Erzählens scheinen so dem skandinavischen Modernismus die Grenzen zu stecken und zu gleicher Zeit das seit jeher Spezifische auf diesen Gebieten zu retten.

\section{AUTEUR}

\section{RÉGIS BOYER}

Université Paris IV - Sorbonne 